\title{
Efectos de la intensificación y el abandono ganaderos en la composición, diversidad y funcionamiento de pastizales mediterráneos
}

\author{
C. Pérez Carmona1 1,* \\ (1) Departamento de Ecología, Facultad de Ciencias, Universidad Autónoma de Madrid, 28049 Madrid, España. \\ * Autor de correpondencia: C. Perez Carmona [carlos.perez@uam.es]
}

> Recibido el 04 de diciembre de 2013, aceptado el 13 de febrero de 2014

Pérez Carmona, C. 2014. Efectos de la intensificación y el abandono ganaderos en la composición, diversidad y funcionamiento de pastizales mediterráneos. Ecosistemas 23(1):73-78. Doi.: 10.7818/ECOS.2014.23-1.13

Los sistemas ganaderos, probablemente el uso del suelo más extendido y el que demanda más recursos a nivel global, han experimentado grandes cambios en las últimas décadas. En Europa, y muy especialmente en el área mediterránea, se observa un proceso simultáneo de intensificación y abandono, que compromete la diversidad y la capacidad de proveer servicios de estos sistemas. Históricamente, el pastoreo ha estado muy estrechamente ligado a los ecosistemas mediterráneos, que han soportado la actividad de los herbívoros domésticos durante milenios. Puesto que el pastoreo se considera un factor esencial para la conservación de estos sistemas, es de especial interés conocer cuáles son las consecuencias de los procesos de intensificación y abandono. La presente tesis doctoral tiene como principal objetivo describir los efectos que el proceso dual de intensificación y abandono ganadero tiene sobre la composición, funcionamiento y persistencia de los pastos mediterráneos, centrándose específicamente en el estudio de las dehesas, uno de los ejemplos más representativos de estos ecosistemas.

Puesto que el pastoreo afecta a la vegetación a través de diversos mecanismos (Dobarro et al. 2013), y dado que sus efectos se manifiestan a distintos niveles, el abordaje de este objetivo requiere examinar distintos aspectos de manera simultánea. Por ello, la tesis está dividida en tres bloques, ordenados en un orden de detalle decreciente, y que se centran respectivamente en efectos a nivel de especie, de comunidad y de paisaje. Con excepción del capítulo centrado en los efectos del abandono de la trashumancia, realizado en dehesas al Norte de la provincia de Jaén, todos los trabajos que componen esta tesis se han llevado a cabo en el área de la finca de Moncalvillo (San Agustín de Guadalix, Madrid, 40 $38^{\prime}$ $\mathrm{N} ; 3^{\circ} 70^{\prime} \mathrm{W}$; $860 \mathrm{~m}$ de altitud media), una dehesa de encinas (Quercus ilex ssp. ballota) y enebros (Juniperus oxycedrus) con pastos en los que abundan las especies anuales.

\section{Efectos del pastoreo en las especies}

Aunque muchas especies vegetales aparecen preferentemente en zonas pastoreadas (especies increaser) o no pastoreadas (especies decreaser), se sabe muy poco acerca de los efectos que los distintos procesos -defoliación, pisoteo y deposición de heces y orina- tienen en las distintas especies. Para estudiar el posible efecto de la deposición de heces, se realizó un experimento en el que se examinaron los efectos de los lixiviados de excrementos de vaca en la germinación de semillas y desarrollo inicial de plántulas de 14 especies anuales con distinta respuesta al pastoreo (Carmona et al. 2013a). Los resultados mostraron que la simple presencia de lixiviados, independientemente de su concentración, redujo significativamente la germinación y desarrollo inicial de las plántulas de especies decreaser, mientras que no afectó a las increaser (Fig. 1). Esta respuesta no lineal sugiere que los lixiviados favorecen una mayor actividad de patógenos que afectaría a ambos tipos de especies de distinta manera. Los lixiviados provenientes de excrementos se perfilan como uno de los factores que pueden estar detrás de las distintas respuestas al pastoreo.

\section{Cambios a nivel de comunidad: diversidad taxonómica y funcional}

De entre los componentes de la diversidad biológica, la diversidad funcional es el que está más fuertemente asociado a los procesos ecosistémicos, y por lo tanto a la provisión de servicios (Hooper et al. 2005). En muchas ocasiones se asume una correlación positiva entre riqueza de especies y diversidad funcional, lo que implica que las reducciones en la riqueza de especies asociadas a los cambios de usos del suelo causan una reducción similar en la diversidad funcional. Sin embargo, esta relación no es universal, y varía entre distintos sistemas y/o circunstancias (Mayfield et al. 2010). El estudio de la relación entre diversidad taxonómica y funcional puede, por lo tanto, aportar una importante información acerca de los efectos que los cambios de uso del suelo pueden tener en el funcionamiento de los ecosistemas. En un primer paso, la comparación entre fincas pastoreadas y abandonadas nos permitió observar que, aunque el abandono del pastoreo no tuvo ningún efecto en la riqueza de especies, sí afecto de forma importante a la composición funcional de las comunidades de pastos. En concreto, se produjo un incremento de especies con alto SLA y con semillas de tamaño grande, y una disminución en la cobertura de especies de altura baja, con porte rastrero o postrado o con floración temprana (Peco et al. 2012). Además se observó un descenso en la fertilidad de los suelos tras el abandono ganadero, muy posiblemente causado por reducciones en las tasas de reciclado de nutrientes y en la descomponibilidad de la hojarasca. 


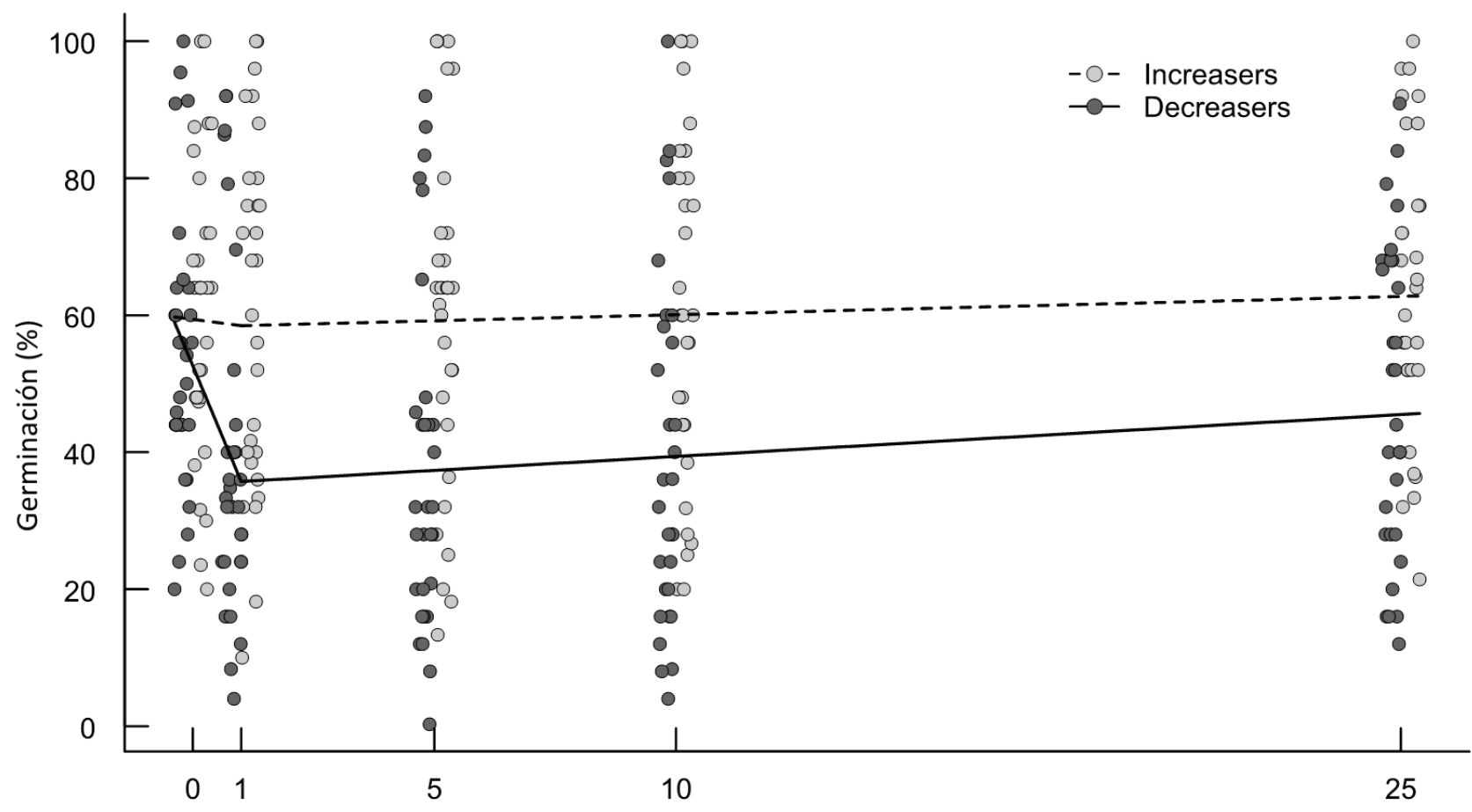

Concentración del lixiviado

Figura 1. Efecto de la concentración del lixiviado de excrementos en los porcentajes de germinación de especies con distinta respuesta al pastoreo (7 especies increaser y 7 decreaser), analizado por medio de una regresión por partes con un umbral al 1 \% de concentración.

A continuación se estudió la estabilidad temporal -comparando un año húmedo y un año seco- de los patrones de composición y diversidad funcional a lo largo de gradientes de pastoreo y de disponibilidad de recursos. Los resultados obtenidos muestran que los patrones de composición funcional y riqueza funcional (Mason et al. 2005) difieren significativamente entre distintos caracteres funcionales, hábitats y niveles de pastoreo, pero son bastante estables entre años (Fig 2).

Por el contrario, los patrones de divergencia funcional fueron mucho más variables en el tiempo, sugiriendo una mayor importancia de la competencia por la luz en los años húmedos. Asimismo, se confirmó que el efecto del pastoreo depende fuertemente de la disponibilidad de recursos (de Bello et al. 2006). Así pues, mientras que altas intensidades de pastoreo redujeron la diversidad taxonómica y funcional en condiciones secas, en condiciones de alta disponibilidad hídrica el pastoreo produjo un aumento de la diversidad taxonómica, pero no afectó a la funcional (Carmona et al. 2012). Aunque los hábitats más secos sufrieron grandes cambios interanuales en su diversidad taxonómica, su diversidad funcional permaneció estable entre años, lo que indica que ambos aspectos de la diversidad no tienen por qué estar acoplados (Fig. 3).

Al abordar el estudio de los cambios interanuales en los patrones de diversidad funcional, esta tesis abre una nueva vía para el análisis de los patrones de convergencia y divergencia asociados a cambios en la disponibilidad de recursos, que hasta ahora se habían ceñido al estudio de gradientes espaciales. Las diferencias interanuales en disponibilidad hídrica pueden afectar significativamente a los procesos primarios que estructuran las comunidades de plantas anuales, por lo que sus efectos sobre la diversidad funcional deberían considerarse en futuros estudios.

\section{Escala de paisaje: teledetección y efectos del abandono de la trashumancia}

Los procesos de intensificación también son importantes a escala de paisaje. El problema del sobrepastoreo se está acentuando en las zonas en las que hay una mayor concentración de ganado, tanto a nivel de finca -puntos de agua o establos-como a nivel regional, debido al abandono de la trashumancia. En el último bloque de la tesis se abordaron estos problemas, primero, con la presentación de una metodología basada en técnicas de SIG y teledetección para facilitar la gestión ganadera de sistemas ambientalmente heterogéneos como los pastizales mediterráneos. Esta aproximación reveló de nuevo la importancia de la disponibilidad de recursos como moduladora del efecto del pastoreo. Mientras que en zonas húmedas la capacidad de recuperación de la vegetación se maximizó a niveles intermedios de presión ganadera, en zonas secas esta capacidad disminuyó de manera consistente a medida que aumentó la presión ganadera (Carmona et al. 2013b). La integración de herramientas de teledetección, SIG y "data mining" (Fig. 4) permitirá mejorar la gestión ganadera al mejorar sensiblemente nuestra capacidad de predecir los efectos de distintas acciones.

Finalmente, se estudiaron los efectos del abandono de las practicas trashumantes en la regeneración y el estado vegetativo del arbolado en dehesas de encina. Para ello, se compararon fincas con ganado trashumante -pastoreadas sólo seis meses al año-y fincas con ganado estante (Carmona et al. 2013c). A pesar de las diferencias en carga ganadera entre ambas prácticas, la temporalización del pastoreo tuvo un importante efecto en la densidad y condición vegetativa de las encinas (Fig. 5). Así pues, el abandono de practicas ganaderas tradicionales como la trashumancia aparece como una de las razones que pueden estar detrás del problema de la falta de regeneración del arbolado en las dehesas. 


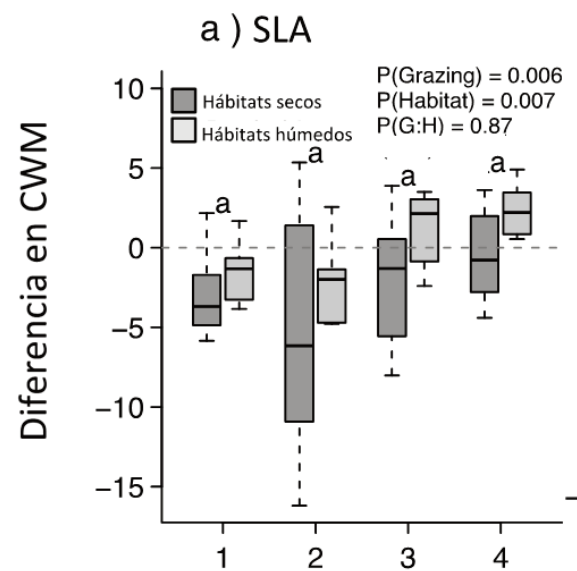

b ) Altura

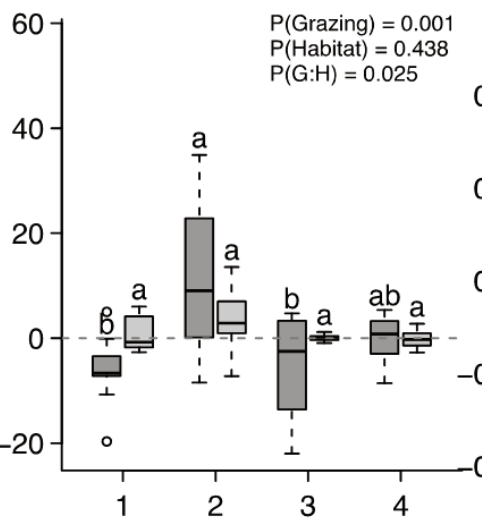

c) Peso de semilla
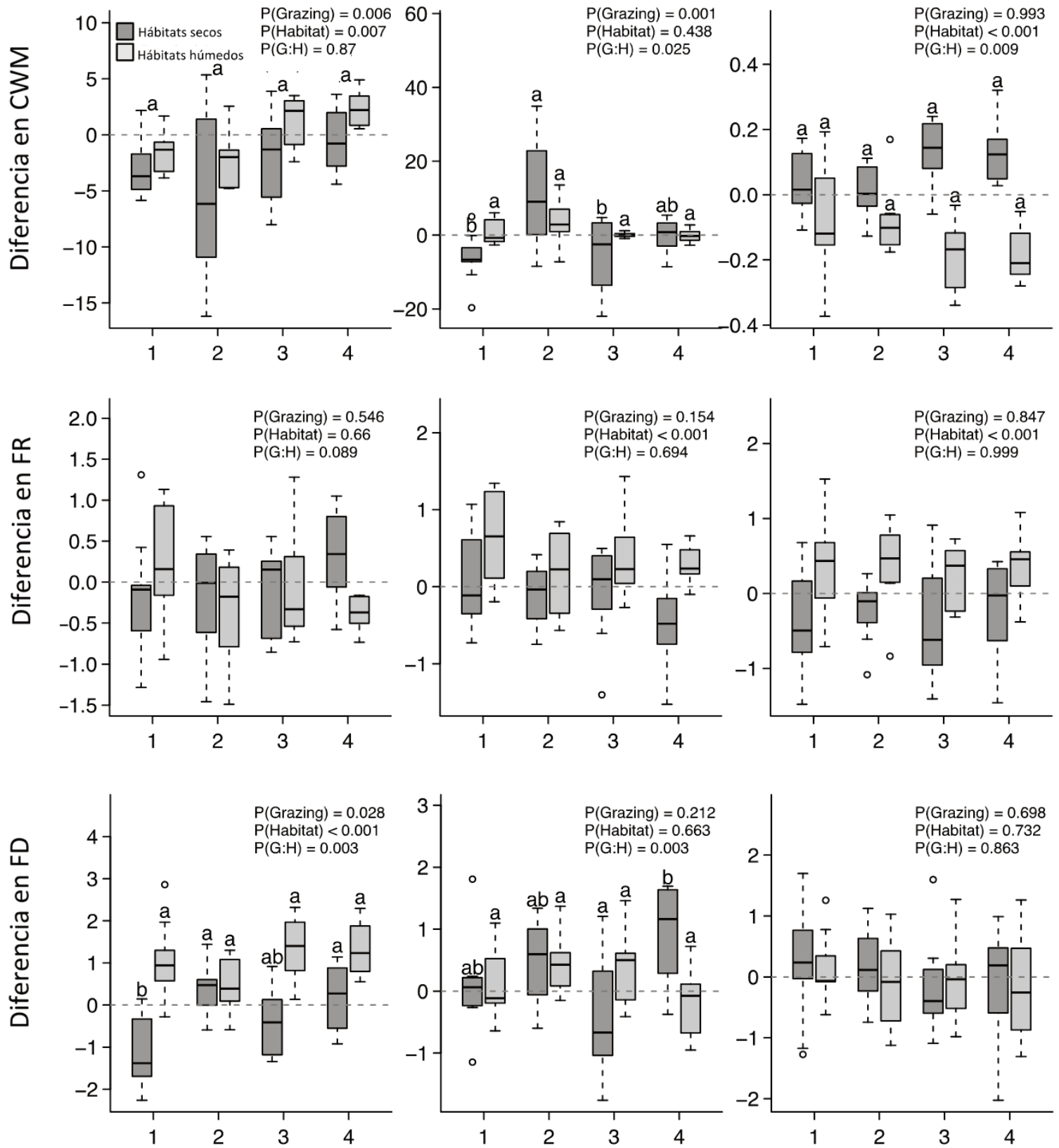

Intensidad de pastoreo

Figura 2. Efecto de la intensidad del pastoreo y el tipo de hábitat en la variación interanual en los valores promedio (CWM; paneles superiores), riqueza funcional (FR; paneles intermedios) y divergencia funcional (FD; paneles inferiores) de los distintos caracteres funcionales estudiados. El cambio fue calculado restando al valor del año húmedo (2010) el del año seco (2009) en cada sitio. En cada panel se muestran los p-valores del pastoreo (grazing), tipo de hábitat y su interacción (G:H), obtenidos por medio de ANOVAs. Cuando G:H es significativa, diferentes letras indicas diferencias significativas entre niveles de pastoreo (Test de Tukey, $p<0.05$ ). Cuando G:H no es significativa, pero el pastoreo sí, diferentes letras indican diferencias significativas entre niveles de pastoreo, combinando ambos hábitats (Test de Tukey, $p<0.05$ ). 


\section{a) Año seco}
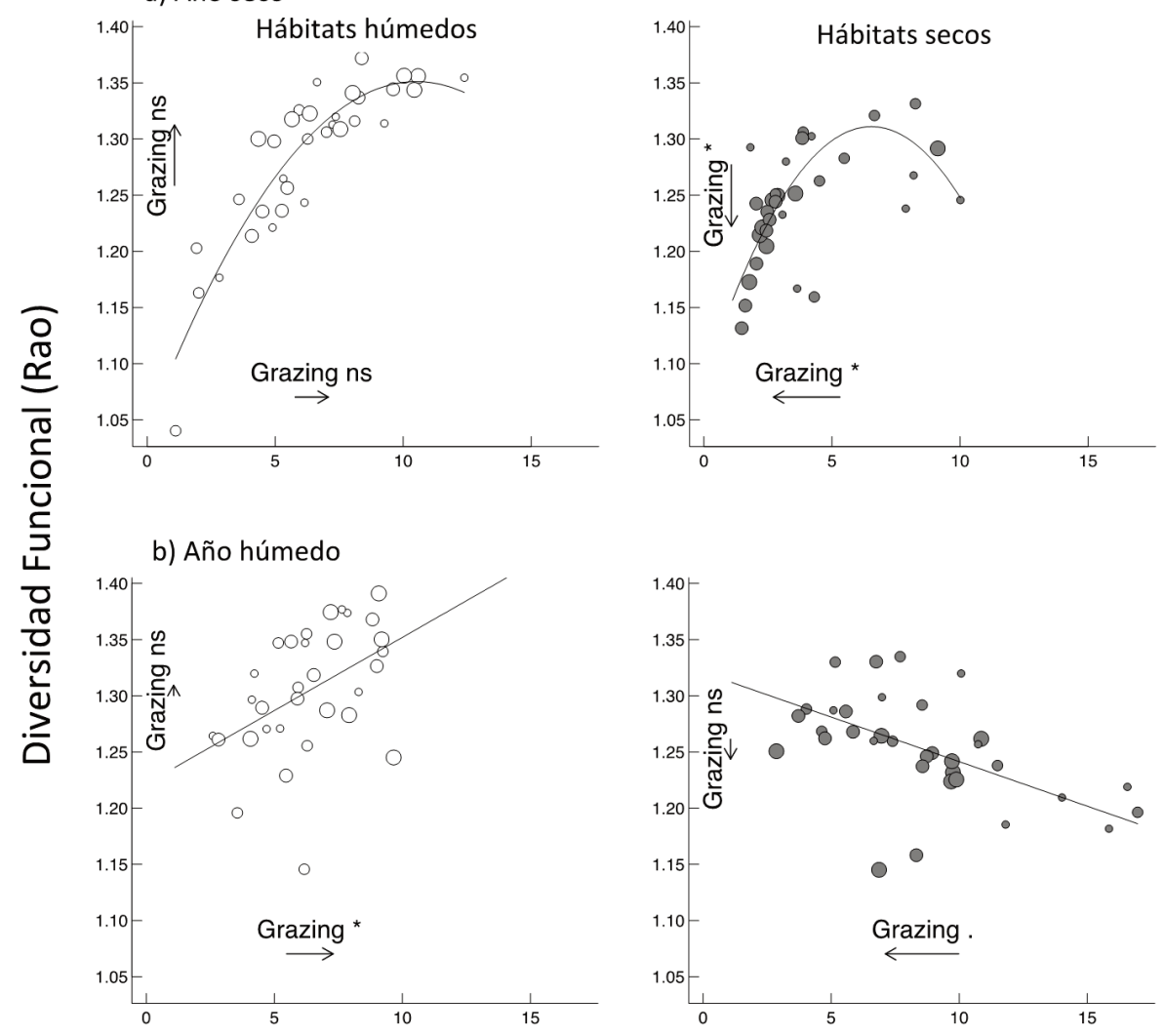

Diversidad Taxonómica (Simpson)

Figura 3. Relación entre la diversidad taxonómica y la funcional (basada en cuatro caracteres funcionales: SLA, altura, peso de semilla y clonalidad) en dos hábitats y años con marcadas diferencias de productividad. La flechas dibujadas en cada panel indican el efecto del pastoreo sobre la diversidad taxonómica (flechas horizontales) y funcional (flechas verticales), empezando en el nivel de pastoreo menos intenso y terminando en el más intenso y su nivel de significación, analizado por medio de regresiones lineales (ns: no significativo; $\bullet: p<0.1 ;$ *: $p<0.05$ ).

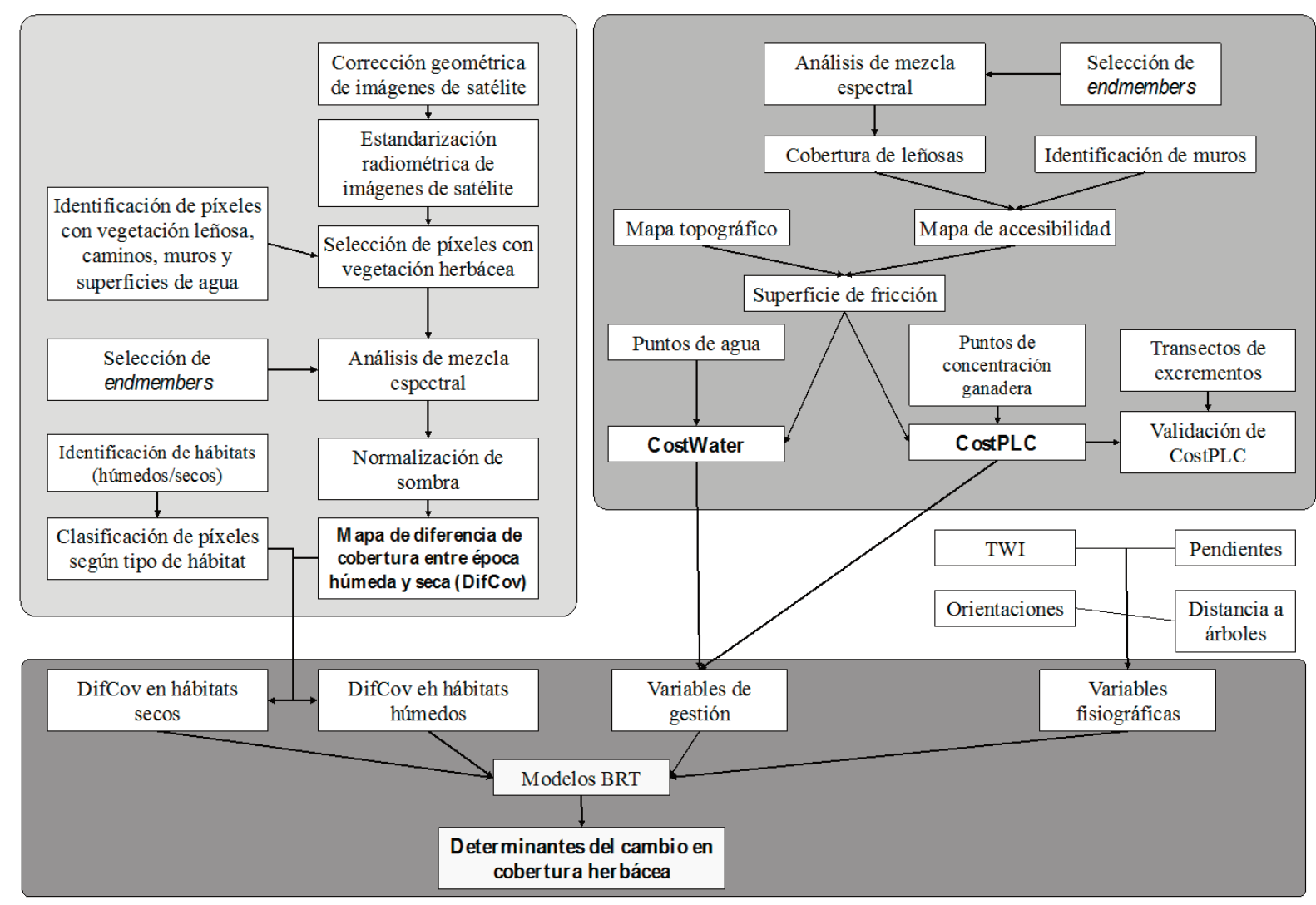

Figura 4. Flujo de trabajo seguido para estudiar los determinantes del cambio de cobertura de vegetación herbácea entre la época húmeda (primavera) y la seca (verano). 
Índice de intrincamiento

Brotes comidos (\%)
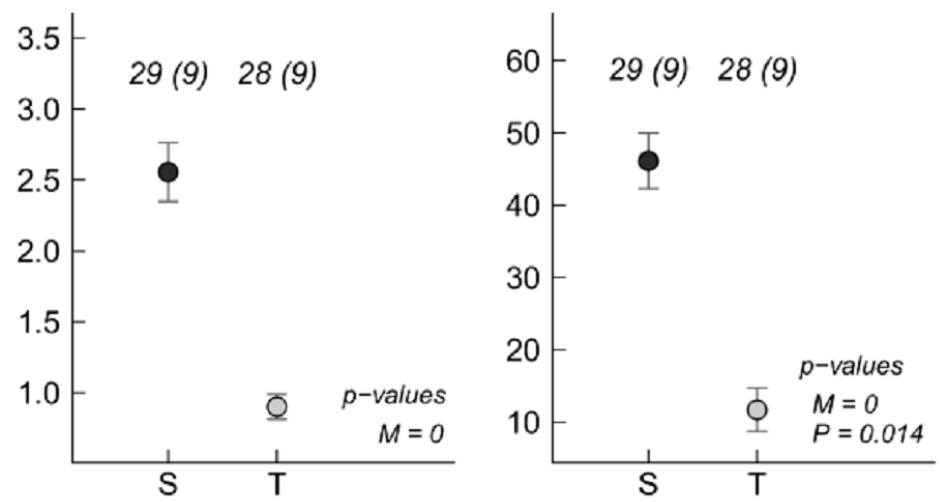

Superficie cubierta (\%)

Diámetro $(\mathrm{cm})$

Altura $(\mathrm{cm})$
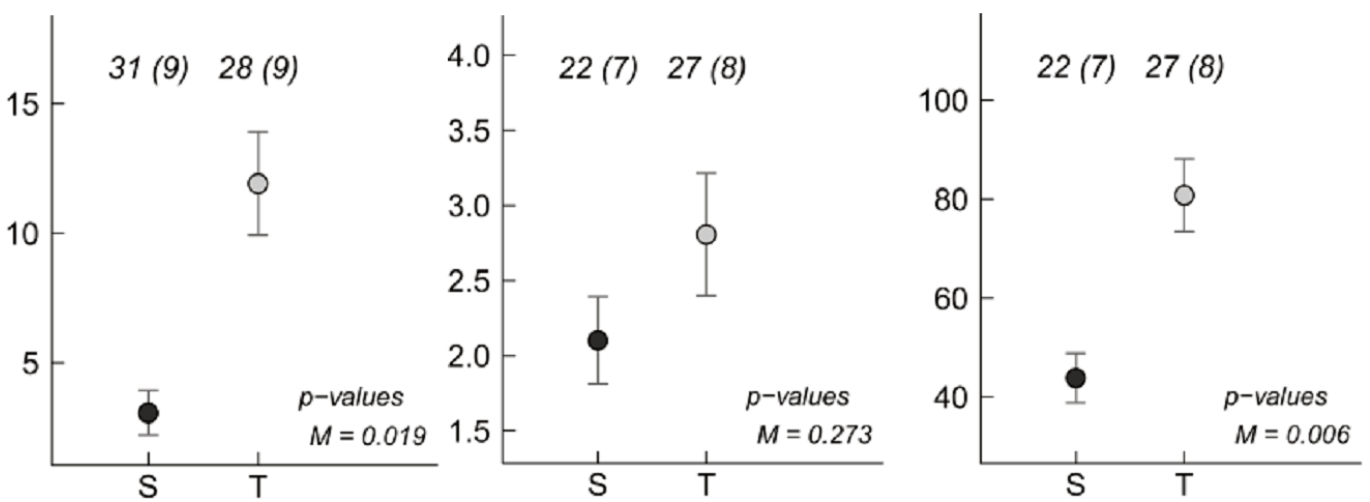

Pequeñas (pies/ha)

Medianas (pies/ha)
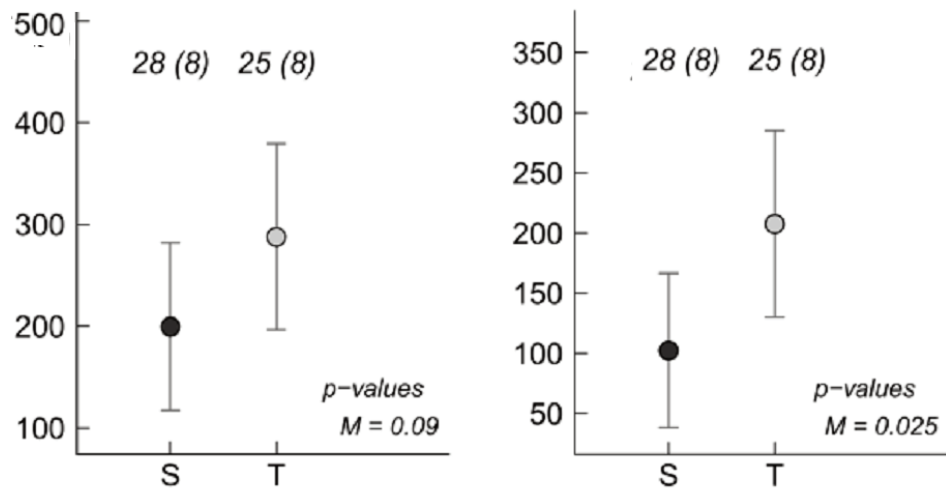

Grandes (pies/ha)

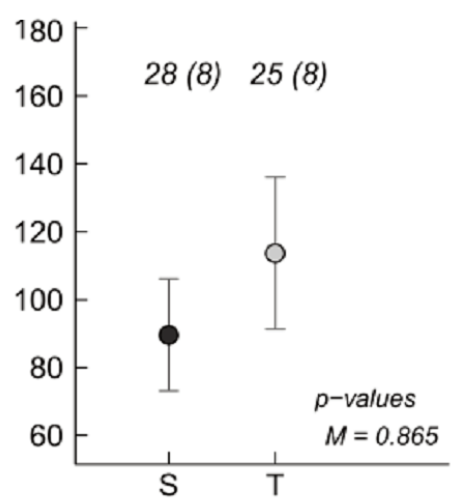

Figura 5. Efecto del tipo de manejo (S: estante; T: trashumante) en los diferentes indicadores del estado de las encinas (indicadores de ramoneo en paneles superiores, indicadores de regeneración en los intermedios e indicadores de estructura del arbolado en los inferiores). Las barras de error representan la media \pm 1 error estándar. Para cada variable, se muestran los p-valores de los predictores tipo de manejo (M) y carga ganadera (P; sólo se muestra en caso de tener un efecto significativo). Encima de cada media se muestra el número de parcelas y el número de fincas (entre paréntesis) en los que se midieron las variables. 


\section{Agradecimientos}

Esta tesis doctoral ha sido financiada por los proyectos CGL2007-63382 y CGL2011-24871 del MINECO, por el Ministerio de Educación (Beca FPI BES-2008-009821) y por el Programa REMEDINAL2 de la Comunidad de Madrid (S-2009/AMB/1783).

\section{Referencias}

Carmona, C.P., Azcárate, F.M., de Bello, F., Ollero, H.S., Lepš, J., Peco, B. 2012. Taxonomical and functional diversity turnover in Mediterranean grasslands: interactions between grazing, habitat type and rainfall. Journal of Applied Ecology 49: 1084-1093.

Carmona, C.P., Azcárate, F.M., Peco, B. 2013a. Does cattle dung cause differences between grazing increaser and decreaser germination response? Acta Oecologica 47: 1-7.

Carmona, C.P., Röder, A., Azcárate, F.M., Peco, B. 2013b. Grazing management or physiography? Factors controlling vegetation recovery in Mediterranean grasslands. Ecological Modelling 251: 73-84.

Carmona, C.P., Azcárate, F.M., Oteros-Rozas, E., González, J.A., Peco, B. 2013c. Assessing the effects of seasonal grazing on holm oak regene- ration: Implications for the conservation of Mediterranean dehesas. Biological Conservation 159: 240-247.

de Bello, F., Lepš, J., Sebastiá, M.T. 2006 Variations in species and functional plant diversity along climatic and grazing gradients. Ecography 29: 801-810.

Dobarro, I., Carmona, C.P., Peco, B. 2013. Dissecting the effects of simulated cattle activity on floristic composition and functional traits in Mediterranean grasslands. PLoS One 0079822.

Hooper, D.U., Chapin, F.S., Ewel, J.J., Hector, A., Inchausti, P., Lavorel, S. et al. 2005. Effects of biodiversity on ecosystem functioning: a consensus of current knowledge. Ecological Monographs 75: 3-35.

Mason, N.W.H., Mouillot, D., Lee, W.G., Wilson, J.B. 2005. Functional richness, functional evenness and functional divergence: the primary components of functional diversity. Oikos 111: 112-118.

Mayfield, M.M., Bonser, S.P., Morgan, J.W., Aubin, I., McNamara, S., Vesk, P.A. 2010. What does species richness tell us about functional trait diversity? Predictions and evidence for responses of species and functional trait diversity to land-use change. Global Ecology and Biogeography 19: 423-431.

Peco, B., Carmona, C.P., de Pablos, I., Azcárate, F.M. 2012. Effects of grazing abandonment on functional and taxonomic diversity of Mediterranean grasslands. Agriculture, Ecosystems and Environment 152: 27-32.

\section{CARLOS PÉREZ CARMONA}

\section{Effects of grazing intensification and abandonment on the composition, diversity and functioning of Mediterranean grasslands}

\section{Tesis Doctoral}

Departamento de Ecología. Facultad de Ciencias, Universidad Autónoma de Madrid

Noviembre de 2012

Directores: Begoña Peco Vázquez y Francisco Martín Azcárate

\section{Publicaciones resultantes de la tesis}

Carmona, C.P., Azcárate, F.M., de Bello, F., Ollero, H.S., Lepš, J., Peco, B. 2012. Taxonomical and functional diversity turnover in Mediterranean grasslands: interactions between grazing, habitat type and rainfall. Journal of Applied Ecology 49: 1084-1093.

Carmona, C.P., Azcárate, F.M., Peco, B. 2013a. Does cattle dung cause differences between grazing increaser and decreaser germination response? Acta Oecologica 47: 1-7.

Carmona, C.P., Röder, A., Azcárate, F.M., Peco, B. 2013b. Grazing management or physiography? Factors controlling vegetation recovery in Mediterranean grasslands. Ecological Modelling 251: 73-84.
Carmona, C.P., Azcárate, F.M., Oteros-Rozas, E., González, J.A., Peco, B. 2013c. Assessing the effects of seasonal grazing on holm oak regeneration: Implications for the conservation of Mediterranean dehesas. Biological Conservation 159: 240-247.

de Bello, F., Carmona, C.P., Mason, N.W.H., Sebastia, M.T., Lepš. J. 2013. Which trait dissimilarity for functional diversity: trait means or trait overlap? Journal of Vegetation Science 24: 807-819.

Peco, B., Carmona, C.P., de Pablos, I., Azcárate, F.M. 2012. Effects of grazing abandonment on functional and taxonomic diversity of Mediterranean grasslands. Agriculture, Ecosystems and Environment 152: 27-32 